\title{
El Neuromarketing y las emociones, factor de éxito en la construcción de marcas en los emprendimientos
}

\author{
Neuromarketing and emotions, a success factor in \\ brand building in entrepreneurship
}

Emma Mendoza Vargas Universidad Técnica Estatal de Quevedo Quevedo - Ecuador emendoza@uteq.edu.ec

Jhon Boza Valle

Universidad Técnica Estatal de Quevedo Quevedo - Ecuador jboza@uteq.edu.ec

Harold Escobar Terán Universidad Técnica Estatal de Quevedo

Quevedo - Ecuador hescobar@uteq.edu.ec

Galo Macías España Universidad Técnica Estatal de Quevedo

Quevedo - Ecuador gmacias@uteq.edu.ec

Revista Cumbres Vol.5 №1

Versión impresa ISSN 1390-9541

Versión electrónica ISSN 1390-3365

http://investigacion.utmachala.edu.ec/revistas/index.php/Cumbres 


\title{
RESUMEN
}

La industria hotelera en los últimos años se ha desarrollado significativamente, al igual que la demanda de esta investigación que tiene como objetivo determinar el empleo del Neuromarketing y las emociones para garantizar el éxito en la construcción de marcas en los emprendimientos de la ciudad e Quevedo. A través de la revisión bibliográfica se determina que el Branding, disciplina orientada a la medición de las reacciones irracionales que viabilizan una relación emocional con el consumidor, debido a que capta la atención cerebral, refuerza la experiencia personal en la satisfacción de las necesidades. La metodología empleada para la investigación es de carácter cualitativo y se utilizó una muestra intencional de 100 emprendimientos de la ciudad de Quevedo afiliados a la Cámara de Comercio. En este sentido la investigación denota que los emprendimientos de la ciudad de Quevedo, emplean el neuromarketing para conocer a los consumidores y desarrollar otros importantes procesos de mercadeo. En esa aplicación influyen positivamente los conocimientos que sobre esta disciplina poseen los emprendedores, lo cual hace que en la construcción de la marca considere el estímulo emocional de los clientes para generar una conexión que viabilice el posicionamiento de sus negocios, a pesar de su aplicación, su generalización se encuentra limitada por cuestiones de costo y desconocimiento.

Palabras clave: Neuromarketing, emociones, construcción de marca, emprendedores.

\begin{abstract}
This research aims to determine the use of Neuromarketing and emotions to ensure success in the construction of brands in the endeavors of the city and Quevedo. Through the literature review it is determined that Branding, a discipline oriented to the measurement of irrational reactions that make possible an emotional relationship with the consumer, because it captures brain attention, reinforces personal experience in the satisfaction of needs. The methodology used for the research is of a qualitative nature and an intentional sample of 110 enterprises from the city of Quevedo affiliated to the Chamber of Commerce was used. In this sense, the research shows that the enterprises of the city of Quevedo use Neuromarketing to get to know consumers and develop other important marketing processes. In this application, the knowledge that entrepreneurs have about this discipline has a positive influence, which means that in the construction of the brand, consider the emotional stimulus of the clients to generate a connection that makes possible the positioning of their businesses, despite their application, its generalization is limited by questions of cost and ignorance.
\end{abstract}

Keywords: Neuromarketing, emotions, brand building, entrepreneurs. 


\section{INTRODUCCIÓN}

Los emprendimientos necesitan insertarse en el entorno empresarial, por lo que las innovaciones deben figurar como un aspecto esencial en su dinámica. El posicionamiento competitivo no puede ser una utopía para estos negocios, por lo tanto, es necesario que se actualicen los procesos para lograr dar respuesta a las necesidades del consumidor de forma efectiva. Los clientes han de ser la inspiración de las transformaciones encaminadas a ocupar espacios en el mercado nacional e internacional. Es por ello que se requieren de estrategias de mercadeo que propicien activaciones sensoriales en los compradores de un producto o receptores de un servicio (Stacho, Stachová, \& Hudáková, 2015).

El neuromarketing es una herramienta que permite esa conexión. El uso de sus técnicas genera información importante y valiosa para las áreas de mercadeo, ya que investiga los procesos cerebrales de los consumidores, puntualizando en los elementos que conllevan a la decisión de compra. Se consideran los procesos cognitivos y emocionales que propician las actuaciones de los clientes ante una determinada oferta.

Es así que su empleo en el mundo empresarial poco a poco ha dejado de ser una añoranza, sin embargo, su generalización no es una realidad, debido a la intervención de varios factores que se relacionan con su costo, con la ética, con el conocimiento y con los procesos de actualización que se implementan en algunos negocios (Canales, 2013; Monge \& Fernández, 2011). Quizás por ello, cerca del $80 \%$ de todos los nuevos productos no alcanzan resultados positivos durante los primeros tres años de introducción en el mercado (Roth, 2013). Como causa de ese comportamiento puede reconocerse que las alineaciones de las nuevas ofertas con los consumidores no son muy fuertes. Pero, el Neuromarketing contribuye a modificar esta situación al investigar los procesos cerebrales, al demostrar que la mayoría de las acciones humanas se desencadenan de forma inconsciente.

A pesar de que se conoce cómo se generan las decisiones de compra de los sujetos, la práctica del Neuromarketing destaca en grandes empresas y es una alternativa muy poco explotada en los emprendimientos (Sánchez, 2013), obviándose así sus beneficios para poder entrar en el corazón de los consumidores y posicionarse en el mercado. Aunque a nivel internacional destaca esta tendencia, es necesario no caer en generalización y conocer del empleo de las técnicas de la neurociencia en un entorno empresarial concreto.

Por ello, se investiga a los emprendimientos de la ciudad de Quevedo con el objetivo de determinar el empleo del neuromarketing y las emociones para garantizar el éxito en la construcción de marcas. Para dar respuesta a este interés se realiza una breve revisión bibliográfica que permite fundamentar las variables en estudio y una investigación de campo para conocer los conocimientos y el nivel de incorporación del neuromarketing en este entorno empresarial. 


\section{Neuromarketing y emociones}

De acuerdo a la premisa de que las decisiones de los individuos se generan de manera ilógica e inconsciente, el neuromarketing se concentra en el estudio de los procesos y los estímulos cerebrales del consumidor (Morin, 2011; Duque, 2014). La información obtenida guía las estrategias que buscan reforzar la relación entre las empresas y los consumidores. Su ámbito de aplicación es muy abarcador, debido a que puede emplearse para el posicionamiento del producto, para la construcción de una marca (branding), para la fijación del precio y para todos los otros procesos que permiten la inserción y la permanencia en el mercado, hacer frente a la competencia y dar respuestas a las exigencias de los clientes.

Para Braidot (2009) y Klinčeková (2016), con su aplicación es posible conocer los estímulos que se deben considerar para alcanzar impacto, la frecuencia de repetición de una campaña y los estímulos sensoriales que se deben satisfacer con un producto o servicio. También es posible establecer la estrategia más idónea frente al precio y la forma en que se debe organizar y orientar el branding

La investigación y obtención de la información sobre el consumidor se logra a través de la aplicación de técnicas de la neurociencia (la resonancia magnética funcional por imagen, el electroencefalograma y magnetoencefalograma). Su empleo propicia determinar las reacciones de los clientes ante específicos estímulos comerciales porque se identifican las zonas cerebrales que se activan cuando ocurre el contacto con un determinado producto o atributo de una oferta (Ariely \& Berns, 2010; Monge \& Fernández, 2011).

El Neuromarketing permite conocer las motivaciones de compra de forma más precisa que las técnicas tradicionales de investigación de mercado. Su centralidad en los patrones inconscientes del comportamiento y en la toma de decisiones de compra ha permitido reafirmar que los consumidores no actúan de forma racional y que toda elección es una respuesta al nivel de engarce que una empresa logra con sus clientes (Laros \& Steenkamp, 2005).

Por tanto, la finalidad mayor es identificar las necesidades de los clientes, para en función de ello implementar acciones que beneficien la conexión con los consumidores. A través del Neuromarketing se superan desconocimientos sobre las verdaderas exigencias, al no obviarse los procesos internos y metaconscientes (Braidot, 2009; Morin, 2011).

En el caso de la construcción de una marca o branding, el neuromarketing permite medir las reacciones irracionales que viabilizan una relación emocional con el consumidor. Si con anterioridad se otorgaba importancia a complejos componentes visuales de una marca; con la generalización de las técnicas de las neurociencias es posible conocer y poner en práctica que todos elementos o atributos de una marca deben ajustarse a lo que realmente llama la atención de los clientes y genera reacciones positivas. La memorización lejos de ser un resultado de la repetición, requiere de vínculos emocionales, por lo cual, se deben aplicar el neuromarketing para conocer qué aspectos o elementos activan esa relación (Ladhari, 2009; Ozgen \& Duman, 2012). 
Según Pop et al. (2009), el entendimiento de las causas de la motivación de los seres humanos, será la regla de oro del futuro. Por lo tanto, el ámbito empresarial debe centrarse en la comprensión de la actuación irracional de los consumidores. Sin embargo, en la actualidad continúa la centralidad en la producción de mercancías ajenas a la forma en que se produce el comportamiento de los clientes y a los factores que motivan una determinada elección y que permiten forjar una relación emocional muy beneficiosa para la fidelización.

En una primera instancia, "la emoción puede ser entendida como el resultado de una evaluación de la medida en que los objetivos de uno se cumplen en la interacción con el medio ambiente" (Ortony, Clore, \& Collins, 1988). Sin embargo, en el ámbito del marketing, describe las reacciones que se generan cuando ocurren transformaciones fisiológicas y se produce su interpretación subjetiva (Coca, 2010).

Aunque son numerosas las mediciones de las reacciones emocionales, desde el ámbito de las ciencias sociales se ha considerado que resulta imposible evaluarlas. No obstante, la aplicación de técnicas de la neurociencia ha permitido conocer su relación con el sistema límbico. Sobre todo, el rol de la amígdala y la región del lóbulo frontal del cerebro en la gestión de las emociones positivas, mientras que el hemisferio derecho tiene mayor participación en la gestión de las emociones negativas (Derbaix \& Poncin, 2005). Todas esas reacciones se pueden identificar y aplicar desde el neuromarketing.

De acuerdo a Klaric (2015), la aplicación del neuromarketing permite construir y posicionar una marca porque ha permitido conocer que se debe dar importancia a la mente y no a la gente, a partir de la puesta en práctica de lo siguiente:

Tabla 1: Claves del Neuromarketing para la construcción de una marca

\begin{tabular}{|c|c|}
\hline Claves & Impacto \\
\hline $\begin{array}{l}\text { Prestar } \\
\text { atención a los } \\
\text { ojos de los } \\
\text { usuarios }\end{array}$ & $\begin{array}{l}\text { La mirada de los consumidores no miente, por lo tanto, es esencial conocer qué es lo } \\
\text { que capta su mirada y hacia dónde la dirige. Se trata de comprender el interés de los } \\
\text { clientes hacia componentes visuales y verbales. }\end{array}$ \\
\hline $\begin{array}{l}\text { Otorgar } \\
\text { importancia a } \\
\text { lo sencillo }\end{array}$ & $\begin{array}{l}\text { Lejos de resaltar que lo complejo es lo que despierta reacciones en los consumidores, } \\
\text { se ha demostrado que lo más fácil conecta con mayor precisión con los clientes, a mayor } \\
\text { sencillez más capacidad de recuerdo se presenta, porque se generan mayores estímulos } \\
\text { sensoriales con lo básico. }\end{array}$ \\
\hline $\begin{array}{l}\text { La imagen se } \\
\text { completa en } \\
\text { el cerebro }\end{array}$ & $\begin{array}{l}\text { Se otorga relevancia a la capacidad cerebral de imaginar lo que no se observa o que se } \\
\text { desea que se observe. Por tanto, es necesario motivar expectativas y ello implica que no } \\
\text { se muestre o se transmita todo lo que se desea comunicar. }\end{array}$ \\
\hline $\begin{array}{l}\text { Emplear } \\
\text { formas orgá- } \\
\text { nicas }\end{array}$ & $\begin{array}{l}\text { Se ha comprobado que existe mayor estimulación cerebral cuando se recurre a formas } \\
\text { que se relacionan con los elementos de la naturaleza. De ahí que las formas redondas } \\
\text { generen mayor sensación de bienestar. }\end{array}$ \\
\hline $\begin{array}{l}\text { Recurrir a las } \\
\text { imágenes y a } \\
\text { las metáforas }\end{array}$ & $\begin{array}{l}\text { Emplear estos recursos garantiza interpretaciones cerebrales positivas, debido a que se } \\
\text { relacionan los productos con las imágenes que ya están en el subconsciente. }\end{array}$ \\
\hline $\begin{array}{l}\text { Forjar víncu- } \\
\text { los emociona- } \\
\text { les y generar } \\
\text { buen humor }\end{array}$ & $\begin{array}{l}\text { Se han de crear estrategias que promuevan la relación para que el cerebro se abra a los } \\
\text { estímulos profundos que motivan el recuerdo hacia una determinada marca. }\end{array}$ \\
\hline
\end{tabular}


Desde una perspectiva similar, pero insistiendo en otras claves, Peters (2002) y Arroyo (2015) señalan que para conquistar al consumidor cada uno de los procesos de construcción de la marca debe captar toda la atención cerebral de los consumidores, reforzar su experiencia y establecer sólidos vínculos emocionales con la marca. De ahí que se hace necesario conocer qué es lo que estimula la aceptación de un producto, lo que genera interpretaciones negativas y lo que motiva a la compra.

De lo expuesto hasta el momento puede concluirse que con el empleo del neuromarketing es posible lograr una mayor conexión con los consumidores, al comprender sus procesos cerebrales y establecer estrategias que potencien reacciones positivas. El estudio y estímulo del subconsciente de los clientes permite gestionar de forma más efectiva una marca, a partir de la generación de un vínculo emocional.

\section{Marca}

Como ya se ha planteado, una eficiente gestión de marca requiere de conocer el comportamiento de los consumidores. Con esta información se logra mayor efectividad en el nombre, en el símbolo o en el diseño de los bienes o servicios de un negocio y una mayor diferenciación de similares ofertas (Kotler, 2001). Quiere ello decir, que desde el momento inicial será posible enfocar la imagen de acuerdo a las necesidades de los clientes y lograr el posicionamiento deseado para la marca y su promoción.

Si con anterioridad se identifican las posibles reacciones, es posible que ocurra de forma exitosa la toma de decisiones sobre las particularidades, atributos y la personalidad de la marca. En todo ese proceso de construcción se han de seguir las recomendaciones de Peters (2002) y Zara \& Tuta (2013) quienes consideran que el éxito de una marca se relaciona con la pasión y las emociones que se logren despertar.

Por tanto, se han de emplear estímulos significativos para lograr la seducción y el engarce de los órganos sensoriales de los consumidores, para que de forma inconsciente se generen reacciones positivas y la marca quede en los mecanismos metaconscientes de los clientes. La creación de acciones que buscan motivar múltiples sentidos arrojará mayores impactos, porque conlleva a la generación de lo que se conoce como un romance de marca, es decir, al placer, la excitación y al dominio (Patwardhan \& Balasubramanian, 2011).

Según lo mencionado por estos autores (Patwardhan \& Balasubramanian, 2011), surge una vez que el cliente tiene un efecto positivo fuerte y alto en la marca, la marca causó una gran excitación y el cliente tuvo plena conciencia de la marca. Esto se llama respuesta al apego emocional del cliente que causa un afecto positivo hacia la marca.

Por otro lado, se conoce que cuando la intimidad, la pasión y el compromiso existen en una marca, se contribuyen fuertemente a la lealtad hacia el objeto (Albert, Merunka, \& Valette-Florence, 2008). Estos tres componentes contribuirán a la fidelización y se expresarán hacia el comportamiento de compra (Ismail \& Spinelli, 2012). 
La investigación científica (Azlina \& Mohd, 2015; Al Pop, Dabija, \& Iorga, 2014) ha demostrado que el posicionamiento de la marca no es resultado de las iniciativas que se desarrollan en el ámbito empresarial. La percepción y definición que realizan los consumidores constituyen aspectos decisivos para lograr insertarse, escalar y mantenerse en el mercado. Todo ello es resultado de los procesos cerebrales inconscientes, por lo que es necesario conocer esas reacciones de forma previa para enfocar las estrategias y poseer mayor posición en la gestión.

Así se optimizan las técnicas y recursos del branding, debido a que es posible estimular sensorialmente al consumidor, pues se conoce la relación existente entre los procesos cerebrales y las actuaciones de los clientes. De esta forma, es posible disminuir los riesgos empresariales, por el lanzamiento de productos o servicios que no dan respuesta precisa a las necesidades de los consumidores, por lo cual su aplicación en los emprendimientos forjaría un trayecto hacia el éxito.

\section{MATERIALES Y MÉTODOS}

El estudio realizado presentó un enfoque mixto, es decir, se empleó un procedimiento cuali-cuantitativo que es "igual a mayor amplitud, profundidad, diversidad, riqueza interpretativa y sentido de entendimiento" (Hernández, Fernández y Baptista, 2006). Para ello se tomó una muestra intencional de 100 emprendedores de Quevedo, afiliados a la Cámara de Comercio de esta ciudad. Con la revisión bibliográfica fue posible fundamentar teóricamente el tema y a través de una investigación de campo, donde se aplicó la encuesta, su determinó el empleo del neuromarketing y las emociones para garantizar el éxito en la construcción de marcas en los emprendimientos de la ciudad de Quevedo. Luego de suministrado el cuestionario, los métodos deductivos e inductivos permitieron identificar los problemas y realizar una discusión en base a los resultados de investigaciones previas.

\section{RESULTADOS Y DISCUSIÓN}

A continuación, se presenta y analiza la información recopilada durante la investigación de campo.

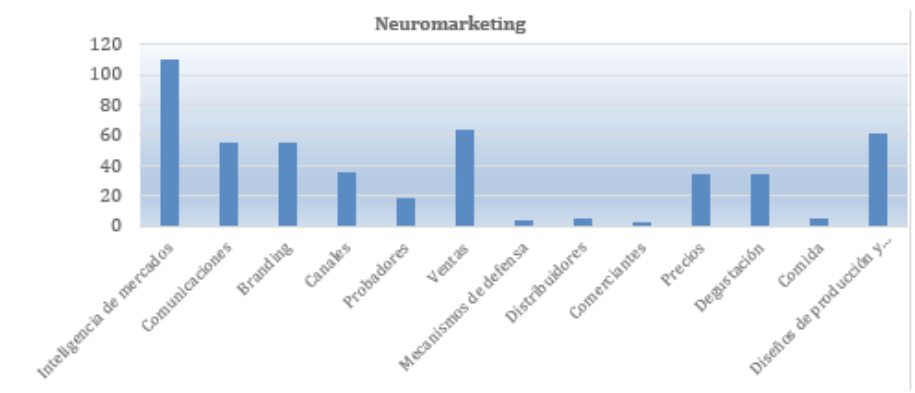

Figura 1. Aplicación del Neuromarketing 
Aunque las respuestas de los emprendedores fueron muy diversas (figura 1), puede reconocerse que poseen conocimientos sobre las aplicaciones del neuromarketing. Principalmente, señalaron que es funcional para la inteligencia de mercados, para el diseño de productos y servicios, para las ventas y en el branding. Esto es favorable para su aplicación en la construcción de marca, ya que los procesos parten de un conocimiento previo sobre el valor de esta disciplina.

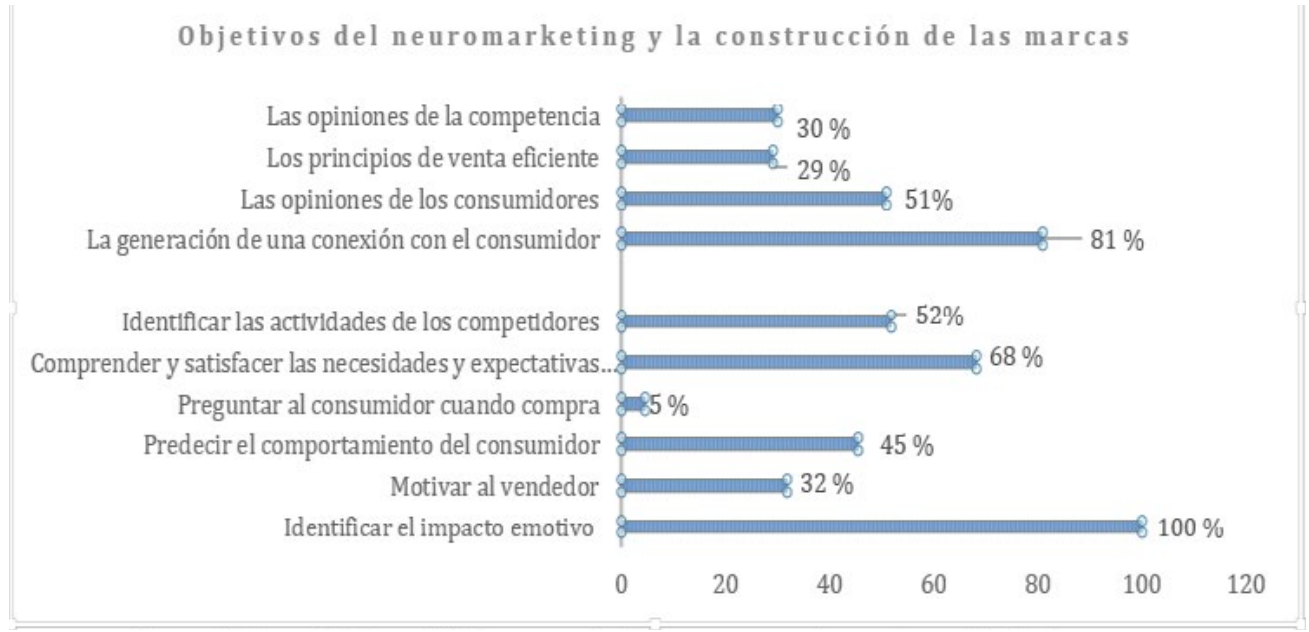

Figura 2. Los objetivos del Neuromarketing y su uso en la construcción de las marcas

Los emprendedores de la cuidad de Quevedo conocen los objetivos de esta disciplina. Si bien dan mayor importancia al impacto emotivo, dominan cada una de las finalidades del Neuromarketing (figura 2). Esto corrobora que los miembros de la muestra se encuentran actualizados y capacitados en temas muy beneficios para el desarrollo de la innovación y de los negocios. El éxito de la marca de los emprendimientos investigados responde a la generación de una conexión con el consumidor y a la consideración de sus opiniones. Con estas respuestas se evidencia que en los negocios de la ciudad se innovan y actualizan los procesos, al combinar procedimientos tradicionales con la novedad del Neuromarketing.

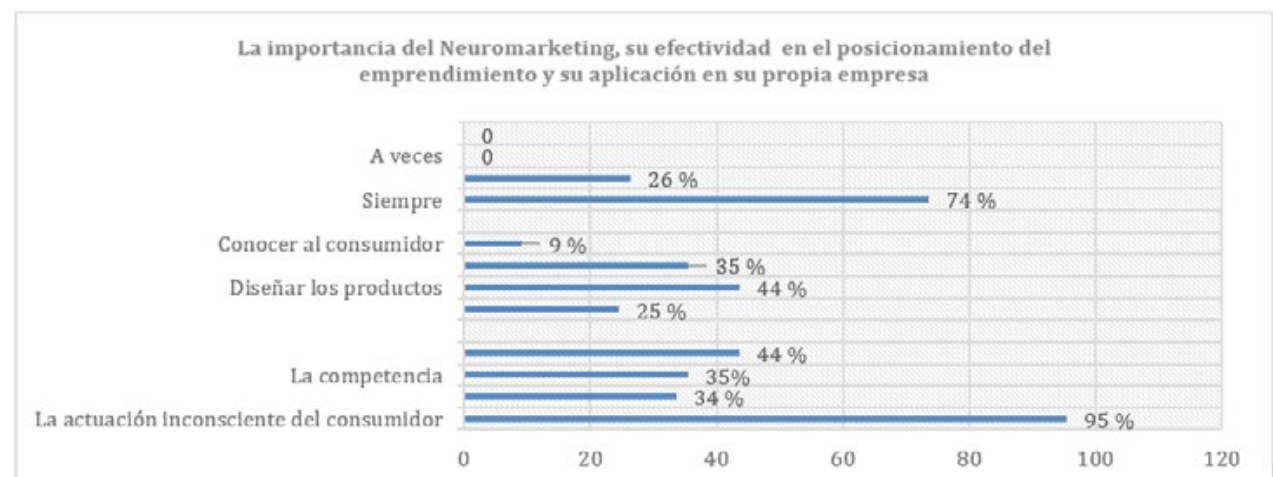

Figura 3. Importancia, efectividad en aplicación del Neuromarketing en los emprendimientos 
Coincidiendo con los postulados teóricos de esta disciplina, para los emprendedores la actuación inconsciente del consumidor es el interés del Neuromarketing, evidenciándose así la preparación que se posee en torno al tema (figura 3). Este conocimiento puede definirse como una fortaleza al momento de aplicar esta disciplina para un área específica del marketing.

De acuerdo a la información recopilada, los emprendimientos de la ciudad de Quevedo han empleado algunas de las herramientas del Neuromarketing para conocer al consumidor, fundamentalmente, con lo cual se evidencia que se ha generalizado una actualización de la investigación de mercado. Llama la atención que, en menor medida, su aplicación ha beneficiado a la construcción de la marca, lo cual puede estar influyendo en su posicionamiento y en la fidelización.

Los emprendedores que conformaron la muestra de estudio son conscientes de que para posicionar sus negocios es necesario aplicar siempre o casi siempre el Neuromarketing, ya que permite entrar en el corazón de los consumidores y diferenciar las ofertas de la competencia, al determinarse lo que necesitan y quieren los consumidores.

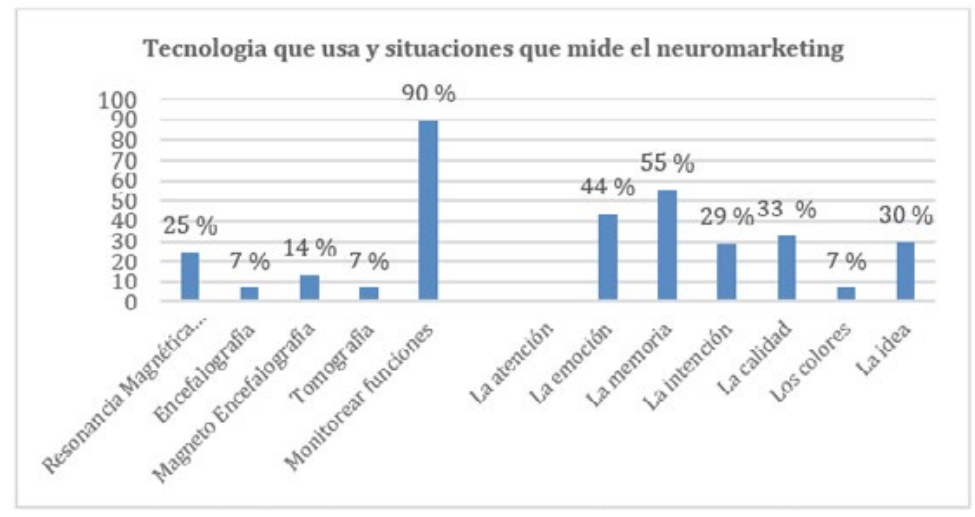

Figura 4. Tecnologías y Neuromarketing de las mediciones

De acuerdo a los emprendedores, el monitoreo de funciones es la técnica que más emplean para construir su marca (figura 4). Esta preferencia puede estar dada por las limitaciones que existen en cuanto al alcance y uso de las demás propuestas. A pesar de ello, los negocios no dejan de buscar soluciones para conocer y poner en práctica todos elementos o atributos de una marca que permitan ajustarse a lo que realmente llama la atención de los clientes y genera reacciones positivas. La centralidad en los elementos que se miden con el Neuromarketing corroboran las competencias de los emprendedores sobre este tema. A su criterio, las evaluaciones se interesan por la atención, la memoria, la calidad y la emoción. 


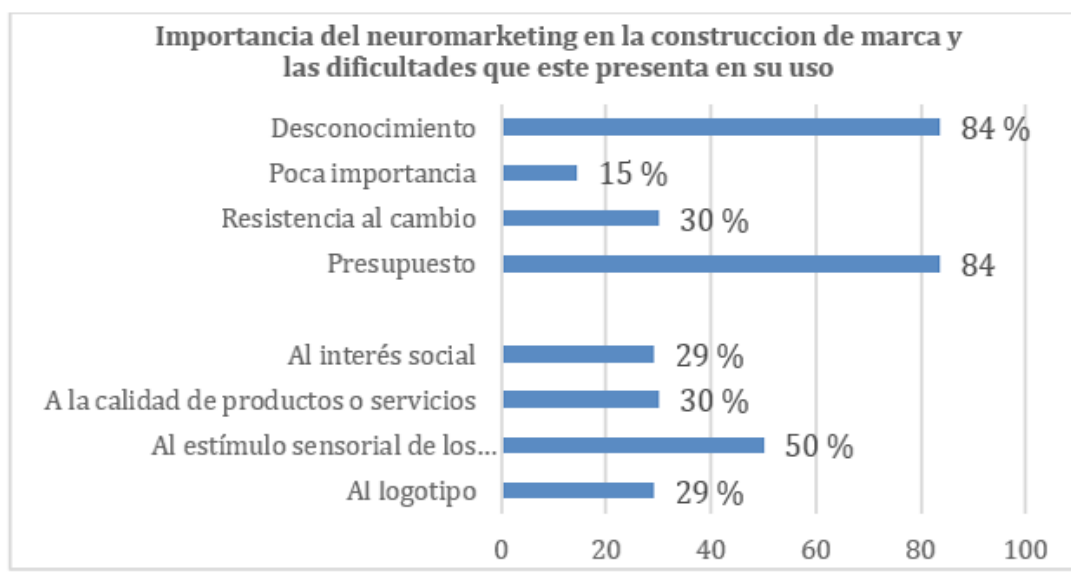

Figura 5. El Neuromarketing, su importancia y dificultades en la construcción de marcas

En la ciudad de Quevedo, los emprendimientos construyen su marca considerando el estímulo sensorial de los consumidores y la calidad de los productos y servicios (figura 5). Así realizan el branding de acuerdo a los principales planteamientos del Neuromarketing, lo cual beneficia la asertividad de la toma decisiones sobre las particularidades, atributos y la personalidad de la marca.

De acuerdo a los emprendedores, aunque conocen del Neuromarketing y lo aplican en la construcción de marca, su empleo se dificulta por el desconocimiento y por sus costos Realmente el uso de las técnicas de la neurociencia es algo costoso, cuando a nivel de empresa, nacional y territorial no se han creado las condiciones, ni las alianzas para su generalización.

Coincidiendo con lo planteado por Morin (2011) y Duque (2014), los emprendedores consideran que el neuromarketing se concentra en el estudio de los procesos y los estímulos cerebrales del consumidor, al momento de entrar en contacto con un atributo comercial u oferta. Como bien manifestaron, con su empleo se da importancia al comportamiento inconsciente del cliente, ya que se ha demostrado que algunas zonas cerebrales se activan cuando ocurre el contacto (Ariely \& Berns, 2010; Monge \& Fernández, 2011). Con la atención a los patrones inconscientes del comportamiento y su relación con la toma de decisiones de compra ha sido posible reafirmar que los consumidores no actúan de forma racional y que toda elección es una respuesta al nivel de engarce que una empresa logra con sus clientes (Laros \& Steenkamp, 2005).

Como bien se reconoció durante la investigación de campo, su empleo es diverso y beneficia a varias áreas de mercadeo, debido a que genera información importante y valiosa sobre los clientes que permite expandir su ámbito de aplicación. Es por ello que en la ciudad de Quevedo se emplea para la inteligencia de mercados, para el diseño de productos y servicios, para las ventas y en el branding.

Para la construcción de la marca de sus negocios, los emprendedores dieron total importancia al estímulo sensorial de los consumidores. Se 
ha demostrado que, a mayor potencia del estímulo emocional, mayor será la conexión neurológica de los clientes con las ofertas del mercado (Domingo, 2009). De acuerdo con Peters (2002) y Arroyo (2015) esto es de suma importancia para conquistar al consumidor. Cada uno de los procesos de construcción de la marca debe captar toda la atención cerebral, reforzar la experiencia y establecer sólidos vínculos emocionales. Todo ello conlleva a una conexión con el consumidor, lo cual se tiene en cuenta, según la investigación de campo, en los emprendimientos encuestados.

Coincidiendo con Klaric (2015), los emprendedores consideraron que siempre la aplicación del neuromarketing permite construir y posicionar una marca. Esto se debe a que los negocios se están enfocando en la mente de los clientes, mediante el empleo de técnicas como el monitoreo funcional. El uso de otras técnicas se imposibilita por sus altos costos y también por desconocimiento como se conoció a través de la investigación de campo.

Este panorama concuerda con el criterio de Canales (2013) y Monge \& Fernández (2011) quienes afirman que la generalización del neuromarketing no es una realidad, debido a la intervención de varios factores como la ética, el conocimiento y los montos de inversión. A pesar de ello, los emprendimientos de la ciudad de Quevedo ya dan pasos, bajo la influencia de esta disciplina, para construir y alcanzar el éxito de sus marcas.

\section{CONCLUSIONES}

El Neuromarketing garantiza la innovación empresarial, la diferenciación y la fidelización de los consumidores en un mercado que se encuentra saturado de productos con muy discretas diferencias. Su aplicación en los emprendimientos propicia que con mayor efectividad se dé respuesta a las necesidades de los clientes, ya que se estudian los procesos mentales para determinar las reacciones de los consumidores ante específicos estímulos comerciales, al conocer las zonas cerebrales que se activan cuando ocurre el contacto con un determinado producto o atributo de una oferta.

En el caso de la construcción de una marca o del branding, el neuromarketing permite medir las reacciones irracionales que viabilizan una relación emocional con el consumidor. Si con anterioridad se otorgaba importancia a complejos componentes visuales de una marca; con la generalización de las técnicas de la neurociencia es posible conocer y poner en práctica que todos los elementos o los atributos de una marca deben ajustarse a lo que realmente llama la atención de los clientes y genera reacciones positivas. Para conquistar al consumidor cada uno de los procesos debe captar toda la atención cerebral de los consumidores, reforzar su experiencia y establecer sólidos vínculos emocionales con la marca. De ahí que se hace necesario conocer qué es lo que estimula la aceptación de un producto, lo que genera interpretaciones negativas y lo que motiva a la compra.

En los emprendimientos de la ciudad de Quevedo se emplea el neuromarketing para conocer a los consumidores y desarrollar otros importantes procesos del mercadeo. En esa aplicación influyen positivamente los conocimientos que sobre esta disciplina poseen los emprendedores, lo cual hace 
que en la construcción de la marca se considere el estímulo emocional de los clientes para generar una conexión que viabilice el posicionamiento de sus negocios. No obstante, llama la atención que a pesar de su aplicación, en su generalización influyen algunas limitaciones de costo y desconocimiento, ante lo cual se requiere de la planeación e implementación de programas de capacitación.

\section{REFERENCIAS BIBLIOGRÁFICAS}

Al Pop, N., Dabija, D. \& Iorga, A. (2014). Ethical Responsibility of Neuromarketing Companies in Harnessing the Market Research-a Global Exploratory Approach. Amfiteatru Economic, 16(35), 26-40.

Albert, N., Merunka, D. \& Valette-Florence, P. (2008). When consumers love their brands: Exploring the concept and its dimensions. Journal of Business Research, 61(10), 1062-1075.

Ariely, D. \& Berns, G. (2010). Neuromarketing: the hope and hype of neuroimaging in business. Revista Nacional de Neurociencia, 11(4), 284-292.

Arroyo, J. (2015). El neuromarketing y la ingeniería del branding. Perfiles de ingeniería, 1(2), 77-83.

Azlina, A. \& Mohd, S. (2015). Development of Neuromarketing Model in Branding Service. Penag: Universiti Sains Malaysia.

Braidot, N. (2009). Neuromarketing en acción. Barcelona: Ediciones Gestión 2000.

Canales, P. (2013). Neuromarketing: ¿El futuro ya està aquí? Empresa, 2, 1-11.

Coca, A. (2010). Neuromarketing: las emociones y el comportamiento de compra. Perspectivas(25), 9-24.

Derbaix, C. \& Poncin, I. (2005). La mesure des réactions affectives en marketing: évaluation des principaux outils. Recherche et Applications en Marketing, 20(2), 55-75.

Duque, E. (2014). Neuromarketing: Una nueva frontera. Suma de Negocios, 5(12), 75-83.

Hernández, R., Fernández, C. \& Baptista, P. (2006). Metodología de la investigación. Iztapalapa. Mexico D. F.: Mc Graw Hill.

Ismail, A. \& Spinelli, G. (2012). Effects of brand love, personality and image on word of mouth: The case of fashion brands among young consumers. Journal of Fashion Marketing and Management, 14(4), 386-398.

Klaric, J. (2015). Véndele a la mente, no a la gente. Barcelona: Paidós.

Klinčeková, S. (2016). Neuromarketing-research and prediction of the future. International Journal of Management Science and Business Administration, 2(2), 53-57.

Kotler, P. (2001). Dirección de marketing. México: Prentice Hall.

Ladhari, R. (2009). Service quality, emotional satisfaction, and behavioural intentions. A study in the hotel industry. Managing Service Quality, 19, 308331.

Laros, J. \& Steenkamp, J. (2005). Emotions in consumer behaviour: a hierarchical approach. Journal of Business Research, 58, 1437-1445(58), 1437-1445.

Monge, S. \& Fernández, V. (2011). Neuromarketing: Tecnologías, Mercado y 
Retos. Pensar la Publicidad, 5(2).

Morin, C. (2011). Neuromarketing: The New Science of Consumer Behavior. Consumer Culture in Global Perspective(48), 131-135.

Ortony, A., Clore, G. \& Collins, A. (1988). The cognitive structure of emotions. New York: University Press.

Ozgen, O. \& Duman, S. (2012). Pre-recovery and post-recovery emotions in the service context: a preliminary study. Managing Service Quality, 22(6), 592-605.

Patwardhan, H. \& Balasubramanian, S. (2011). Brand romance: a complementary approach to explain emotional attachment toward brands. Journal of Product \& Brand Management, 20(4), 297-308 .

Peters, T. (2002). El meollo del branding. Madrid: Ediciones Nowtilus.

Pop, C., Radomir, L., Maniu, I. \& Zaharia, M. (2009). Neuromarketing - Getting inside the Customers's Mind. Annals of Faculty of Economics, 4(1), 804807.

Roth, V. (2013). The Potential of Neuromarketing as a Marketing Tool. Enschede: Bachelor Thesis Conference.

Sánchez, J. (2013). El neuromarketing en la PYME. Disponible en: https://bit.ly/ 2jYYemU

Stacho, Z., Stachová, K. \& Hudáková, M. (2015). Approach of Companies to Customers as SuitableSource of Incentive to Innovate. Procedia Economics and Finance, 34, 11 - 18., 34, 11 - 18.

Zara, I. \& Tuta, M. (2013). Neuromarketing Research - A Classification and Literature Review. Research Journal of Recent Sciences, 2(8), 95-102. 
